\title{
Bilateral rotator cuff repair utilizing augmentation nine patch grafts over 24 years in one patient with polio paraplegia: A case report
}

\author{
Robert Nirschl ${ }^{1}$, Jarrett Helming ${ }^{1}$, Benjamin Ingram ${ }^{1}$, Mehdi Jafari ${ }^{1}$, Farshad Adib ${ }^{1}$, Skye Donovan ${ }^{* 1,2}$ \\ ${ }^{1}$ Nirschl Orthopedic Center, United States \\ ${ }^{2}$ Department of Physical Therapy, Marymount University, United States
}

Received: August 14, 2016

Accepted: August 30, 2016

Online Published: September 1, 2016

DOI: $10.5430 /$ css.v2n4p41

URL: http://dx.doi.org/10.5430/css.v2n4p41

\begin{abstract}
This case report describes the management of sequential rotator cuff (RC) tears with multiple patch graft augmentation in a polio-paraplegic athlete. The patient underwent these operations over a twenty-four year span from 1986-2010. The patient's case was complicated due to the fact he had more than a dozen upper extremity surgeries during this time period. The grafts used were autograft illiotibial band, followed by porcine xenografts, and finally allograft dermal collagen matrixes. This report describes the advancements in graft technology, and the surgical procedure associated with the grafts. After the most recent surgery, the patient exhibited full range of motion in both shoulders, the ability to perform independent transfers and was able to discontinue pain medication. Our case provides evidence of the success rate of using patch grafts in both frail tissue repairs as well as large cuff defects in a patient who places high demand on his upper extremities for daily activities.
\end{abstract}

Key Words: Rotator cuff repair, Patch graft

\section{INTRODUCTION}

This is a case report of sequential rotator cuff (RC) repairs with patch graft augmentation in the same patient. The patient, a polio-paraplegic athlete, underwent these operations over a twenty-four year span from 1986-2010, with follow-up surgery without patch graft augmentation in the right shoulder in 2013. This case highlights several important points regarding the use of patch grafts in the treatment of a paraplegic athlete. Due to the unique demands place on the upper body by this patient, this case report describes the viability (vascularization and incorporation) of sequential patch grafts and the options available for patch graft material. A literature review using the search string "patch graft and RC" revealed only eleven partially related articles, none specifically on this subject.

\section{Case presentation}

The patient, currently a 65 -year-old male former paraplegic internationally competitive athlete, had complete functional loss of both legs at age 5 as a complication of polio. He subsequently developed his upper body physically and remained athletically active despite complete loss of his lower extremity function. During his wheelchair Para-Olympic career he held 8 world records in shot put with 42 gold medals in international competition. Twice he completed the Boston marathon. This high level competition however was injurious

*Correspondence: Skye Donovan; Email: Skye.donovan@marymount.edu; Address: Marymount University- Department of Physical Therapy, 2807 N Glebe Rd, Arlington, VA 22207, United States. 
to the patient's shoulders (though not unexpected) as paraplegic patients have been shown to have a tenfold increase in RC tears compared to controls as diagnosed by MRI. ${ }^{[1]}$ Given the patient's paraplegic state, regaining full use of his shoulders was paramount to performing both his sport and his activities of daily living.

The patient had a total of nineteen upper extremity operations from 1981-2013. A review of this patient's case was approved by the Marymount University IRB. The first of 9 RC repairs utilizing patch graft augmentation started in 1986, with the last occurring in 2010. In total $5 \mathrm{RC}$ repairs in the right shoulder and 4 in the left shoulder were completed using patch graft augmentation. Over time there were 10 additional surgeries for bilateral elbow tendinosis, elbow osteoarthritis, as well as cubital and carpal tunnel syndromes, and one right shoulder RC repair without patch graft augmentation in 2013.

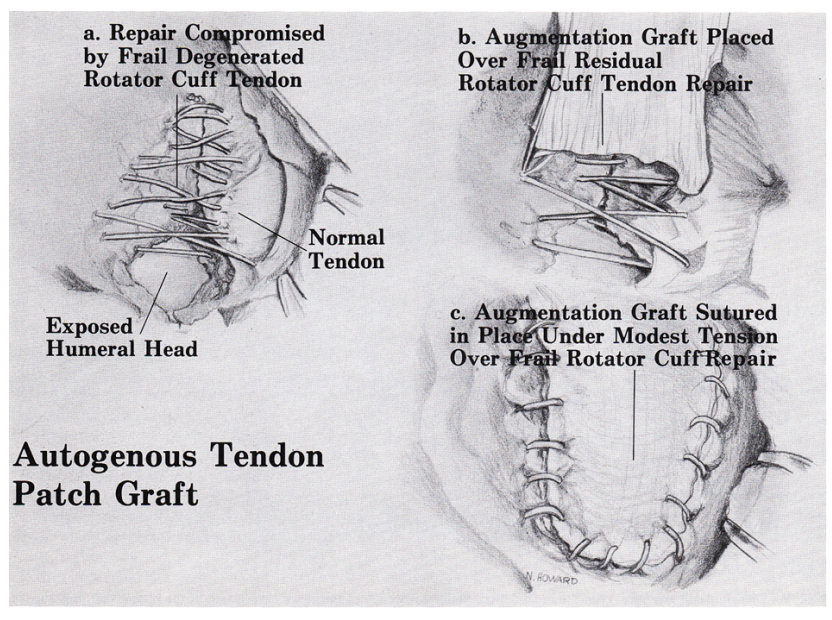

Figure 1. Schematic representation of patch graft technique This Figure shows the patch graft incorporated into frail rotator cuff $(R C)$ repair. This schematic is an accurate representation of the first right shoulder patch graft in the patient of this report. Copied with permission from Rotator Cuff Repair. ${ }^{[2]}$

The first three tendon patch graft augmentations performed in this patient ( 2 right shoulder and 1 left) used portions of autogenous illiotibial band. These patches were harvested at the time of the $\mathrm{RC}$ repair when it was decided that complete and firm repair of the RC could not be accomplished through traditional surgical techniques. The donor sites were a good choice regarding morbidity given the leg disuse secondary to his paraplegia. Despite the absence of developed underlying leg muscles, the illiotibial band itself appeared of reasonable quality. The harvested section of illiotibial band was placed with the fibers in the direction of the supraspinatus tendon over the site of the un-repairable defect. This was then secured with sutures in the medially retracted cuff tendons and corner stitches at the proximal end followed by running parameter stitches towards the greater tuberosity (GT). Initially the distal end was secured by a transosseous suture placed in the GT. The first right shoulder patch graft was found to be secure and covering the entire primary suture repair with smoothness noted in shoulder range of motion. Subacromial smoothing was undertaken but full acromioplasty or resection of the coraco-acromial ligament was avoided (see Figure 1).
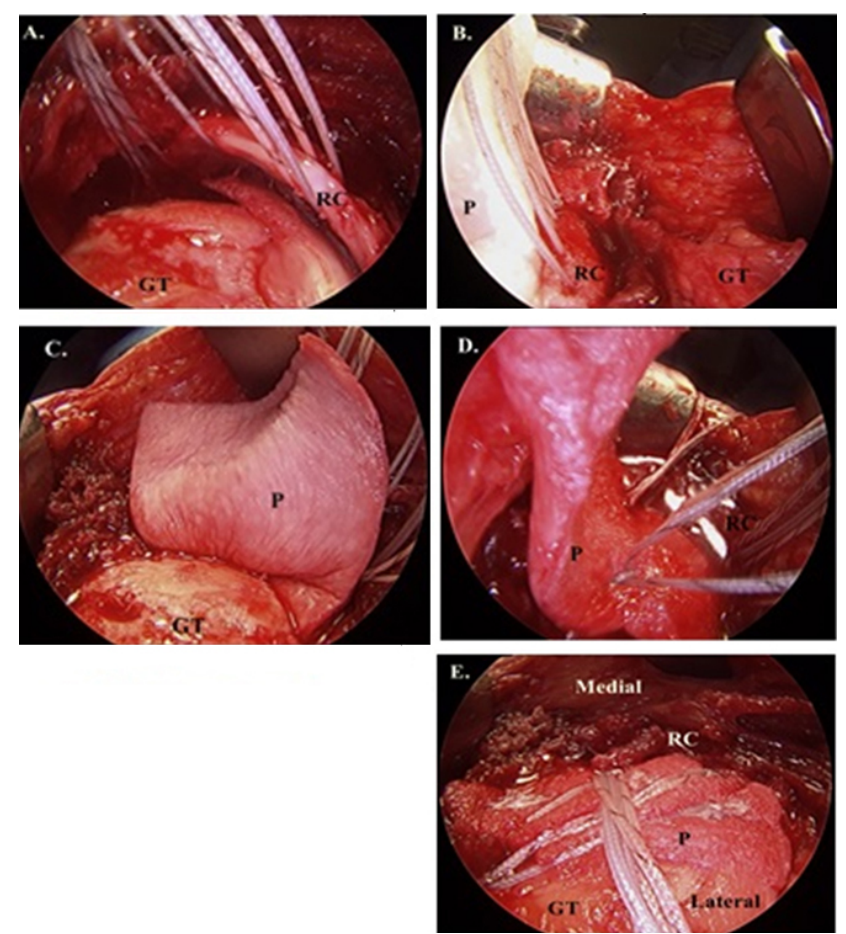

Figure 2. Intraoperative representation of patch graft technique

A. Multiple sutures placed on the lateral edge of retracted rotator cuff $(R C)$, greater tuberosity $(G T)$ exposed;

$B$. Attchment of allograft patch $(P)$ to lateral edge of $R C$ with initial sutures;

$C$. Underside of patch $(P)$ attached to lateralretracted and deficient $R C$, with exposed uncovered GT shown;

$D$. Patch allograft $(P)$ now attached to GT via bone suture anchors, with lateral patch edge still free;

E. Final repair: sutures from medial retratced $R C$ edge, bone suture anchors in mid GT, and lateral row fixation with bone suture anchors (SwiveLock by Arthrex ${ }^{\circledR}$ ).

The function of the coraco-acromial arch was therefore preserved. Subsequent patch graft augmentations evolved from porcine xenografts (one in each shoulder) to dermal allografts (two in each shoulder). Similar operative procedure was used for all subsequent $\mathrm{RC}$ repairs requiring patch graft augmentation but lateral and distal fixation was subsequently secured by bone anchors (see Figures 2 and 3). 


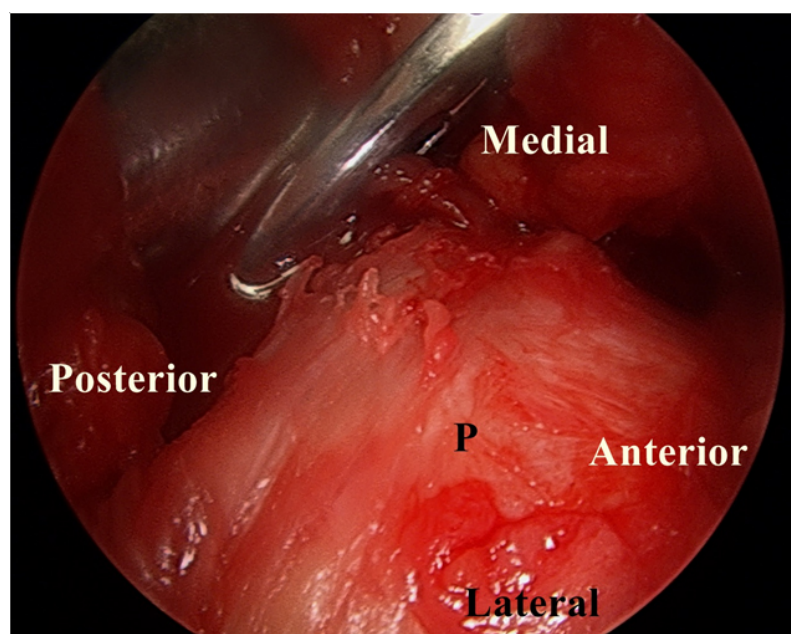

Figure 3. Intraoperative representation of patch graft tissue Intraoperative photo showing incorporated patch graft tissue from previous surgery at the time of the sixth operation (January 29, 2013) in this patient. A new Rotator cuff tear is shown just posterior to the patch graft $(P)$ within the infraspinatous tendon. Tear is shown at the end of the metal probe. A patch was not done at the time of this surgery. Prior incorporated patch $\left(5^{\text {th }}\right.$ patch) was $8 \mathrm{~mm}$ thick at posterior edge.

The patient presented most recently for right shoulder surgery (sixth intervention) on January 29, 2013 with symptoms of recurrent right shoulder pain altering activities of daily living. A recurrent vertical tear was noted at the posterior interface of the patch with the posterior one-half of the infraspinatus. Repair of the vertical tear was undertaken without the need for a patch graft. The prior patches (antero-medial infraspinatus, supraspinatus, and subscapularis regions were fully incorporated, viable, and robust (measuring $8 \mathrm{~mm}$ patch thickness at posterior edge) (see Figure 3).

As noted, as technology developed, autograft illiotibial band, followed by porcine xenografts, and finally allograft dermal collagen matrixes were implanted. The current generation of dermal allografts are thicker and more easily handled. None of the allografts or xenografts resulted in rejection or infection. This patient's graft repairs were performed using an open Saber incision technique by deltoid split. In no surgery was the deltoid detached from the acromion. At the time of the most recent clinical examination on April 26, 2013, the patient, age 65 , weight 250 pounds (mainly upper body obesity), had full motion of both shoulders, power which allowed full body transfers from wheelchair and scooter to bed, the ability to drive a specially equipped van, and no need for pain medications.

\section{Discussion}

The success in this series of operations was return to play at a high level of competition in the early stages of life

Published by Sciedu Press followed by independent performance of activities of daily living (ADL)'s. It should be noted that the authors did not recommend return to sports. Given the large defects in the patient's RC tendons, firm repair would have been compromised without the assistance of patch grafts.

Each subsequent surgical intervention revealed the patch grafts to be incorporated into the native RC. The volume of cuff tissue at the most recent interventions completely covered the humeral head (right shoulder 2005 with 5 total grafts, and one suture repair of the incorporated patch to posterior infraspinatus in 2013, and left shoulder with 4 total grafts, most recently in 2010). Not only had the grafts incorporated but the grafted cuff tissue was thickened. In the Supraspinatus Tendon Articular Side (STAS) region of the right shoulder at last surgery (in 2013), the cuff measured 8 $\mathrm{mm}$ in thickness and the left shoulder (in 2010), measured $9 \mathrm{~mm}$. STAS is defined as the region between the foot print attachment to the GT and the musculotendinous junction. ${ }^{[3]}$

One criticism to this treatment strategy would be that the patient did require repeat procedures as the adjacent RC tissue failed or recurrent tears occurred in, or adjacent to, the patches in succession. Given the need to return to function in this patient for activities of daily living and lifestyle, it can be argued however that the rate of recurrent tears was outweighed by the return to limited sport with ultimate preservation of function into late adult life (age 65 in 2013). With more known now about tensile strengths of various patch graft materials ${ }^{[4,5]}$ and breadth of products available, ${ }^{[6]}$ the selection of patch graft materials would be different today than when the first patch graft augmentation was performed in 1986. Each patch graft material has positive and negative characteristics.

There have been other reports of patch grafting in the literature. The first report that we are aware of was reported in 1978 with the use of freeze-dried fascia lata for the repair of massive RC defects. ${ }^{[7]}$ The most recent publication by Gupta et al. in 2013, describes successful clinical and radiographic outcomes in $73 \%$ of patients who underwent $\mathrm{RC}$ reconstruction with porcine xenograft for 2 tendon irreparable RC tears. ${ }^{[8]}$ In 1986, Ozaki, et al. ${ }^{[9]}$ in Japan reported on repair of massive cuff defects with a polyester grafts. Later carbon filaments were used in repair of massive defects, but this was later abandoned given poor outcomes resulting from the carbon fibers themselves. The senior author's first reports of the use of the patch grafts were in 1988 and 1989. ${ }^{[10,11]}$ Since 1986, others have performed and reported their experience with patch grafts in the RC. Audenaert et al. ${ }^{[12]}$ reported on a case series of 41 patients with Mersilene polyester mesh (Ethicon). Bond 
et al. ${ }^{[13]}$ found 13 of 16 patients had MRI confirmed integration and overall patient satisfaction with the augmentation of irreparable RC tears with GraftJacket Allograft with 2 year average follow-up. One Japanese study by Ito and Morioka ${ }^{[14]}$ compared the McLaughlin procedure alone $(\mathrm{n}=12)$ to repair using a double layer allograft patch $(n=9)$. The McLaughlin procedure described in that article begins with a deltoid split and is essentially a trans-osseous non-tension repair of deficient cuff which clears the articular surface of the humeral head into a trough cut into the medial aspect of the GT just lateral to the humeral head articular surface. This was done because the remaining cuff tissue was insufficient in length to restore normal foot print anatomy. When this procedure could not be accomplished, a patch graft consisting of a double layer of freeze dried allograft fascia lata was placed between the cuff and grove. At mean follow up time 2 years 11 months in the patch graft group, $0 \%$ of the patch graft group experienced clinical evidence of re-tearing while 3 of the 12 patients treated with the regular McLaughlin procedure re-tore within 2 months of the operation. With the three re-tears excluded from the study, the groups were otherwise the same in terms of pain and range of motion after surgery. The decision to use a double patch graft versus a single layer was based on the view of the surgeons that the allograft material (freeze-dried fascia lata from Biodynamics, Germany) was thin in comparison to the RC tendon. Mihata et al. ${ }^{[15]}$ recently described successful patient outcomes utilizing fascia lata autograft and arthroscopic superior capsule reconstruction for those with massive RC tears after nearly 3 years follow-up. This technique reconstructs a superior capsule and attaches to the free edge of the retracted infraspinatus tendon.

Patch grafting is a technique that is best applied to remaining cuff tissue although frail but with some blood supply when full repair cannot be performed. ${ }^{[11]}$ It is the opinion of the lead author that (although less ideal than covering frail tissue which retains some blood supply), performing a patch graft in a massive tear by filling a gap could provide several opportunities even in the absence of cuff tissue (especially the supraspinatus and the inferior aspect of the infraspinatus). The first opportunity would be to create a suspension bridge between the remaining infraspinatus and the subscapularis thus balancing and assisting in their ability to stabilize the humeral head during abduction. The second opportunity would be the potential for re-vascularization of the graft tissue if microfracture were performed in the bare area of the humeral head underneath the patch graft. The final opportunity would be a cushioning effect of the patch and the new tissue ingrowth between the humeral head and the acromion.

The more ideal situation for patch graft incorporation is the covering of frail cuff tissue which has blood supply and is advantageous in the security of the repair as there is more confidence in the post-operative rehabilitation phases. Ito and Morioka in their study in $2003,{ }^{[14]}$ also commented that decreased re-tearing noted on the McLaughlin patch graft group compared to the regular McLaughlin group may be in part due to decreased tension allowed by the patch graft re-enforcement of the primary cuff repair. ${ }^{[14]}$

\section{Comment}

The senior author continues to use patch grafts in both frail tissue repairs as well as large cuff defects in paraplegic and non-paraplegic patients. The current graft of choice is a dermal allograft (see Figures 2 and 3).

\section{Conclusion}

Serial patch graft augmentation during $\mathrm{RC}$ repair allowed this patient to initially continue to compete in international competition, and subsequently function in activities of daily living. For the paraplegic patient, shoulder function is paramount to independent living. The patient currently remains independent in his activities of daily living at age 65.

\section{CONFLICTS OF INTEREST Disclosure}

No financial disclosures or conflicts of interest exist for any of the authors of this report.

\section{REFERENCES}

[1] Akbar M, Balean G, Brunner M, et al. Prevalence of rotator cuff tear in paraplegic patients compared with controls. J. Bone Joint Surg. Am. 2010; 92(1): 23-30. http://dx .doi .org/10.2106/JBJS.H . 01373

[2] Nirschl R. Rotator Cuff Repair. AAOS Instructional Course Lectures (Editor JS Barr). Rosemont, IL; 1989. 447-462 p.

[3] Nirschl R, Davis L, St Pierre P, et al. The supraspinatus tendon articular side lesion "STAS". 2007.
[4] Barber FA, Herbert MA, Coons DA. Tendon augmentation grafts: biomechanical failure loads and failure patterns. Arthrosc. J. Arthrosc. Relat. Surg. Off. Publ. Arthrosc. Assoc. N. Am. Int. Arthrosc. Assoc. 2006; 22(5): 534-538. http://dx.doi.org/10.1016/j.arthr 0.2005 .12 .021

[5] Barber FA, Herbert MA, Boothby MH. Ultimate tensile failure loads of a human dermal allograft rotator cuff augmentation. Arthrosc. J. Arthrosc. Relat. Surg. Off. Publ. Arthrosc. Assoc. N. Am. Int. Arthrosc. Assoc. 2008; 24(1): 20-24. http://dx. doi.org/10.10 
$16 / j \cdot$ arthro. 2007.07 .013

[6] Coons DA, Alan Barber F. Tendon graft substitutes-rotator cuff patches. Sports Med. Arthrosc. Rev. 2006; 14(3): 185-190. http: //dx.doi.org/10.1097/00132585-200609000-00011

[7] Neviaser JS, Neviaser RJ, Neviaser TJ. The repair of chronic massive ruptures of the rotator cuff of the shoulder by use of a freeze-dried rotator cuff. J. Bone Joint Surg. Am. 1978; 60(5): 681-684. PMid: 681390 .

[8] Gupta AK, Hug K, Boggess B, et al. Massive or 2-tendon rotator cuff tears in active patients with minimal glenohumeral arthritis: clinical and radiographic outcomes of reconstruction using dermal tissue matrix xenograft. Am. J. Sports Med. 2013; 41(4): 872-879. http://dx.doi.org/10.1177/0363546512475204

[9] Ozaki J, Fujimoto S, Masuhara K, et al. Reconstruction of chronic massive rotator cuff tears with synthetic materials. Clin. Orthop. 1986; (202): 173-183. http://dx.doi.org/10.1097/0000308 6-198601000-00022

[10] Nirschl R. Rotator Cuff Limited Exposure Repair/reconstruction Utilizing a Patch Graft Technique. Video Journal of Orthopedics. 1998.
[11] Nirschl RP. Rotator cuff surgery. Instr. Course Lect. 1989; 38: $447-$ 462.

[12] Audenaert E, Van Nuffel J, Schepens A, et al. Reconstruction of massive rotator cuff lesions with a synthetic interposition graft: a prospective study of 41 patients. Knee Surg. Sports Traumatol. Arthrosc. Off J. ESSKA. 2006; 14(4): 360-364. http://dx.doi.org/10.1007 /s00167-005-0689-7

[13] Bond JL, Dopirak RM, Higgins J, et al. Arthroscopic replacement of massive, irreparable rotator cuff tears using a GraftJacket allograft: technique and preliminary results. Arthrosc. J. Arthrosc. Relat Surg. Off. Publ. Arthrosc. Assoc. N. Am. Int. Arthrosc. Assoc. 2008; 24(4): 403-409. http: //dx.doi.org/10.1016/j.arthro. 2007 .07 .033

[14] Ito J, Morioka T. Surgical treatment for large and massive tears of the rotator cuff. Int. Orthop. 2003; 27(4): 228-231. http://dx.doi . org/10.1007/s00264-003-0459-4

[15] Mihata T, Lee TQ, Watanabe C, et al. Clinical results of arthroscopic superior capsule reconstruction for irreparable rotator cuff tears. Arthrosc. J. Arthrosc. Relat. Surg. Off. Publ. Arthrosc. Assoc. N. Am. Int. Arthrosc. Assoc. 2013; 29(3): 459-470. http: //dx.doi.org/10.1016/j.arthro.2012.10.022 